\title{
La normativa de los servicios ambientales en sudamérica. Propuestas para una gestión sustentable
}

\author{
Clara María MinAVERRY \\ Instituto de Investigaciones Jurídicas \\ y Sociales Ambrosio Rioja \\ Facultad de Derecho \\ Universidad de Buenos Aires (Argentina) \\ clara.minaverry@gmail.com
}

Recibido: 19 de noviembre de 2013

Enviado a evaluar: 15 de diciembre de 2013

Aceptado: 7 de mayo de 2014

\section{RESUMEN}

El objetivo de este trabajo es analizar los aspectos principales de la normativa sobre servicios ambientales de una selección de países de América del Sur, para poder detectar fortalezas y debilidades y colaborar con posibles propuestas para lograr una gestión sustentable. Metodológicamente se realizó un análisis comparativo de "estudios de caso", y se utilizó el método de observación documental. Los datos cualitativos recogidos fueron secundarios (análisis de registros escritos, tales como doctrina y legislación). Podemos concluir que existe una disparidad en la normativa sobre servicios ambientales de los países de América del Sur que fueron seleccionados. Esta situación va en contra del espíritu del MERCOUSR al que pertenecen Argentina y Paraguay, cuyo objetivo es unificar el marco jurídico ambiental de la región, según surge de la Resolución № 22/92 del Grupo de Mercado Común. Lo mismo ocurre con los países pertenecientes a la Comunidad Andina (Perú y Colombia).

Palabras clave: Servicios ambientales, medioambiente, sustentabilidad, normativa, América del Sur.

\section{Environmental services law in South America. Proposals for a sustainable management}

\begin{abstract}
The purpose of this paper is to analyze the main issues of environmental services regulation from a selection of South American countries, in order to set up strengths and weaknesses and to collaborate with feasible proposals for a sustainable management. Methodologically, we made a comparative analysis of "case studies", and used the documentary observational method. The qualitative data were secondary (written records, such as jurisprudence and regulations). We can conclude that there is a legal disparity in environmental services regulations from the selected countries of South America. This situation goes against the MERCOSUR spirit (where Argentina and Paraguay are members), which main objective is to standardize the environmental legal framework, according to Resolution $\mathrm{N}^{\circ} 22 / 92$ from the MERCOSUR Common Market Group. The same occurs with the countries from the Andean Community (Perú and Colombia).
\end{abstract}

Key words: Environmental services, environment, sustainability, regulations, South America. 


\section{La régulation des services en Amérique du Sud de l'écosystème. Propositions pour une gestion durable}

\section{RÉSUMÉ}

Le but de cet article est d'analyser la réglamentation concernant les services environnementaux dans les pays d'Amérique du Sud sélectionnés, afin de identifier les forces et les faiblesses et de contribuer avec des propositions pour parvenir à une gestión plus soutenable. Du point de vue méthodologique, on a effectué une analyse comparative des "études de cas" et on a utilisé la méthode de l'observation documentaire. Les données qualitatives recueillies ont été secondaires (analyse de documents écrits, tels que la doctrine et la législation). On peut conclure qu'il y a une grande disparité des règles dans la réglementation des services environnementaux dans les pays d'Amérique du Sud qui ont été sélectionnés. Cette situation est contraire à l'esprit du MERCOUSR -l'Argentine et le Paraguay sont États parties- qui a pour but unifier le cadre juridique de la protection de l'environnement dans la región, comme est indiqué dans la Résolution N²2/92 du Groupe Marché Commun du MERCOSUR. La même situation se déroule dans les pays de la Communauté Andine (Pérou et Colombie).

Most clés: Eau, «plan d'eau», l'agriculture, l'Espagne, plans d'action, les politiques de l'eau.

\section{INTRODUCCIÓN}

La figura de los servicios ambientales ha sido desarrollada en diversos países de América del Sur, aunque en algunos con mayor profundidad y desarrollo normativo.

La ley $\mathrm{N}^{\circ} 26.331$ de presupuestos mínimos para la protección ambiental de los bosques nativos de Argentina en su artículo 5, define a los servicios ambientales como "los beneficios tangibles e intangibles, generados por los ecosistemas del bosque nativo, necesarios para el concierto y supervivencia del sistema natural y biológico en su conjunto, y para mejorar y asegurar la calidad de vida de los habitantes".

Esta definición parte de la protección ambiental, con el de que sirva para mejorar la vida de las personas, y por supuesto preservar los recursos naturales para las generaciones futuras (conforme el principio de desarrollo sustentable).

Otro objetivo fundamental de la figura de los servicios ambientales es evitar la deforestación, a través del pago de dinero para que se logren proteger los recursos naturales. Todo esto se encuentra ligado indefectiblemente a la disminución de la pobreza, ya que se resguardan los medios de vida de varias comunidades.

Además, su regulación genera una demanda importante de certificados de servicios ambientales y de esta manera se impulsa la certificación de numerosas superficies por ejemplo de los bosques naturales.

Por su parte el Estado cumple una función primordial ya que brinda diversas herramientas, pero en nuestro caso únicamente evaluaremos las jurídicas.

Particularmente, en Argentina la tendencia observada por parte del Derecho Ambiental es la de proteger legalmente a los recursos hídricos y a los bosques por separado (Minaverry, 2011), salvo en aisladas excepciones normativas.

Una de las cuestiones que dificulta más la aplicación práctica del pago por servicios ambientales es la valoración económica (que es subjetiva y que resulta 
difícil determinarla). Es un aspecto que ha sido ampliamente tratado por diversos organismos internacionales dedicados a la investigación y al diseño de políticas públicas.

Además, para que los programas de pago por servicios ambientales funcionen es necesaria una fuente estable de financiación, y es fundamental identificar adecuadamente a los beneficiarios de los mismos para poder desarrollar un sistema de cobros (Pagiola; Platais, 2002).

La elaboración de una ley específica sobre el pago por servicios ambientales puede conllevar aspectos positivos como negativos.

"La regulación de PSA en una ley especial también ofrece la posibilidad de establecer desde el principio un marco jurídico amplio y adecuadamente estructurado. Esto facilita la compilación, organización y sistematización de las diferentes disposiciones sobre PSA de forma ordenada y adaptada al entorno jurídico y de mercado. Si el desarrollo de una ley es imposible, se podría modificar la legislación medioambiental sectorial vigente. La legislación ambiental sectorial incluye leyes que regulan la protección del medio ambiente y la conservación de la naturaleza en general (como las leyes ambientales generales, las leyes sobre marcos ambientales)" (IUCN, 2010).

Sin embargo, existen diversas herramientas que pueden colaborar para lograr una gestión más sustentable.

\section{OBJETIVO}

Analizar la normativa sobre servicios ambientales de una selección de países de América del Sur, para poder detectar fortalezas y debilidades y colaborar con posibles propuestas para lograr una gestión más sustentable, que han sido implementados por algunas comunidades.

\section{METODOLOGÍA}

Se dividió en las siguientes fases:

a) Fase exploratoria:

Se recopiló doctrina y legislación nacional e internacional, relacionada con la regulación de los servicios ambientales.

b) Fase descriptiva:

La información recogida en la etapa anterior fue clasificada y categorizada, a fin de facilitar su análisis en una etapa posterior, y se dividieron los aspectos positivos y negativos de la normativa de cada país. 
c) Fase analítica:

El análisis de los documentos recogidos en las dos fases anteriores, tuvo por objeto detectar marcos legales que, facilitaron el análisis de las fortalezas y debilidades de los mismos a través del análisis comparativo de los "estudios de caso".

Desde el punto de vista metodológico, se utilizó el método de observación documental.

Los datos cualitativos a recoger fueron secundarios (análisis de registros escritos, tales como doctrina, y legislación).

\subsection{LA REGULACIÓN JURÍDICA DE LOS SERVICIOS AMBIENTALES EN AMÉRICA DEL SUR}

\subsubsection{ARGENTINA}

Actualmente, los servicios ambientales aún no han sido regulados especial e individualmente en la normativa que protege a los recursos naturales de Argentina. Esto coincide con que el desarrollo del Derecho Ambiental local, carece de una vinculación jurídica sistémica ni tendiente a la conservación integral del ambiente.

Sin embargo, uno de los casos excepcionales es el de la ley nacional $\mathrm{N}^{\mathrm{o}} 26.331$ (presupuestos mínimos ambientales para la protección de los bosques nativos), que tiene como objetivo principal el enriquecimiento, la restauración, la conservación, el aprovechamiento y el manejo sostenible de los "servicios ambientales" que brindan los bosques (artículo 1).

Además, esta norma (artículos 30 y 31), establece la creación del fondo nacional para el enriquecimiento y la conservación de los bosques nativos, con el objeto de compensar a las jurisdicciones que conservan los mismos, por los servicios ambientales que éstos brindan (Minaverry, Gally, 2012).

Este Fondo deberá estar integrado por:

a) Las partidas presupuestarias que le sean anualmente asignadas a fin de dar cumplimiento a la presente ley, las que no podrán ser inferiores al $0,3 \%$ del presupuesto nacional;

b) El dos por ciento ( $2 \%$ ) del total de las retenciones a las exportaciones de productos primarios y secundarios provenientes de la agricultura, ganadería y sector forestal, correspondientes al año anterior del ejercicio en consideración;

c) Los préstamos y/o subsidios que específicamente sean otorgados por Organismos Nacionales e Internacionales;

d) Donaciones y legados; 
e) Todo otro aporte destinado al cumplimiento de programas a cargo del Fondo;

f) El producido de la venta de publicaciones o de otro tipo de servicios relacionados con el sector forestal;

g) Los recursos no utilizados provenientes de ejercicios anteriores.

Lo más importante es que el fondo nacional para la conservación de los bosques nativos será distribuido anualmente entre las jurisdicciones que hayan elaborado y tengan aprobado por ley provincial su ordenamiento territorial.

En la Provincia de Buenos Aires puntualmente, se está debatiendo un proyecto de ley provincial, en donde se utilizarán tres categorías que se respetarán, para determinar la intensidad de la protección de los bosques nativos las cuales serán los siguientes:

a) Roja: máxima protección y supondrá un plan de conservación por el cual no se podrá desmontar.

b) Amarilla: dos planes, un plan de conservación y otro de manejo sustentable.

c) Verde: un plan de conservación, uno de manejo y uno de aprovechamiento con cambio de uso de suelo. Se permitiría el desmonte siempre y cuando haya una audiencia pública y un estudio de impacto ambiental que lo apruebe.

También existen otras formas de proteger a los bosques:

a) Red de áreas protegidas con planes de manejo (Reservas nacionales, provinciales, municipales y privadas).

b) Predios de propietarios privados (con aportes a la conservación, acciones de protección y uso sostenible de los recursos).

c) Promoción de protección de los recursos forestales (educación), incentivo de su uso.

Además, en dicha área se han determinado ciertas pautas se va definir si una zona justifica un nivel de protección rojo, amarillo o verde.

• ¿Resguardan márgenes de ríos?

- ¿Implican zonas de conexión entre dos áreas de un mismo ecosistema? 
- ¿Son áreas de alta vulnerabilidad?

• ¿Tienen algún valor sobresaliente?

• ¿Cuál es su potencial productivo?

- ¿Qué uso le otorga la comunidad?

A su vez, la autoridad nacional de aplicación conjuntamente con las autoridades de aplicación de cada una de las jurisdicciones que hayan declarado tener bosques nativos en su territorio, determinarán anualmente las sumas que corresponda pagar, teniendo en consideración para esta determinación:

a) El porcentaje de superficie de bosques nativos declarado por cada jurisdicción;

b) La relación existente en cada territorio provincial entre su superficie total y la de sus bosques nativos;

c) Las categorías de conservación declaradas, correspondiendo un mayor monto por hectárea a la categoría I que a la categoría II.

Luego, la ley $\mathrm{N}^{\circ} 26.331$ (artículo 35) establece que los fondos para la protección de los bosques nativos fueron creados para la protección de bosques nativos, y serán aplicados de la siguiente manera:

a) E1 70\% para compensar a los titulares de las tierras en cuya superficie se conservan bosques nativos, sean públicos o privados; y

b) El 30\% a la autoridad de aplicación de cada jurisdicción, que lo destinará a desarrollar y mantener una red de monitoreo y sistemas de información de sus bosques nativos y a la implementación de programas de asistencia técnica y financiera, para propender a la sustentabilidad de actividades no sostenibles desarrolladas por pequeños productores y/o comunidades indígenas y/o campesinas.

La ley argentina también tiene la intención de excluir de la misma a las comunidades indígenas, ya que en su artículo 2 establece que "quedan exceptuados de la aplicación de la presente ley todos aquellos aprovechamientos realizados en superficies menores a diez hectáreas que sean propiedad de comunidades indígenas o de pequeños productores."

Por eso, la supuesta protección que se incorporó en los artículos anteriores quedó desarticulada para los casos de las comunidades que posean extensiones pequeñas de bosques, las cuales no contarían con el apoyo financiero que brinda la norma, pero habilitándolos para utilizar sus recursos naturales. 
Sin embargo, en Argentina no existen otras normas que hagan referencia al manejo de los servicios ambientales, existiendo un vacío legal que dificulta su implementación en la práctica.

Es importante manifestar, que tal como esta nueva ley establece beneficios y avances en la protección de los bosques nativos en la República Argentina, la misma genera nuevas obligaciones para las diferentes jurisdicciones (provincias y municipios).

Específicamente, en su artículo sexto afirma que se otorga un plazo de un año a partir de la sanción de la ley, para que cada jurisdicción realice un ordenamiento de los bosques nativos existentes en su territorio, de acuerdo a los criterios de sustentabilidad que figuran en el Anexo. La Autoridad Nacional brindará la asistencia técnica, económica y financiera para poder materializar esta obligación.

Cabe aclarar que en el tiempo que transcurra entre la confección del ordenamiento de los bosques nativos y de la sanción de la ley, no pueden autorizarse desmontes.

Por otro lado, en el artículo noveno se fijan tres categorías de conservación, que deben tomarse en cuenta por todas las jurisdicciones al momento de realizar el ordenamiento de los bosques mencionados.

La Categoría I (rojo), se refiere a los sectores de bosques de máxima conservación que de ninguna manera deben transformarse. En esta sección quedarían incluidas las reservas que poseen un valor biológico sobresaliente, y que pueden utilizarse para realizar tareas de investigación científica.

La Categoría II (amarillo) son los sectores de mediano valor de conservación, que con una adecuada restauración podrían ser utilizados como lugares turísticos, de recolección de recursos y también de investigación científica.

En último lugar la Categoría III (verde), son los sectores de bajo valor de conservación que pueden transformarse parcialmente o en su totalidad, siempre siguiendo los criterios establecidos por la normativa de análisis.

Por otra parte, se debe avanzar y definir adecuadamente quién será la autoridad de aplicación de la ley de protección de los bosques nativos.

En el artículo décimo de la ley se establece que será de aplicación el organismo que la Nación, las provincias y la Ciudad Autónoma de Buenos Aires determinen para cada jurisdicción. Luego, en el artículo siguiente establece que para el ámbito Nacional la autoridad de aplicación será la Secretaría de Ambiente y Desarrollo Sustentable de la Nación, o el organismo de mayor jerarquía con competencia ambiental que la reemplace en el futuro.

Asimismo, las Provincias que elaboren y aprueben por ley el ordenamiento territorial de sus bosques nativos, recibirán anualmente fondos para compensar a los titulares de las tierras que conserven los bosques nativos, a través de un aporte no reintegrable que se abonará por hectárea.

Otra temática central de la presente normativa es la relativa a la instrumentación de los desmontes en las diferentes jurisdicciones, que es de mucha importancia para las autoridades de la Secretaría de Ambiente y Desarrollo Sustentable de la Nación. 
La regla general que se establece en la ley es la de que todo desmonte o manejo sostenible de bosques nativos, requerirá necesariamente autorización de la Autoridad Nacional de la jurisdicción a la que corresponda.

Además, se manifiesta que de ninguna manera podrán autorizarse desmontes de bosques nativos correspondientes a las categorías I y II, al igual que la quema a cielo abierto de los residuos derivados de los desmontes.

Sin embargo, se deja abierta la posibilidad para que las personas físicas y jurídicas soliciten autorización para realizar un manejo sostenible de bosques nativos de las categorías I y II. Sin embargo, éstos deberán sujetar su actividad a un Plan de Manejo Sostenible de Bosques Nativos.

También los que soliciten autorización para realizar desmontes de bosques nativos ubicados en la categoría III, deberán adecuar su actividad a un Plan de Aprovechamiento del cambio de uso del suelo.

Además incorpora algunos instrumentos administrativos de política ambiental, que colaboran para lograr una mayor preservación de los bosques nativos.

El primero es el instrumento de la Evaluación de impacto ambiental, siendo que representa un ejercicio de predicción y prevención de una incidencia no deseada en el ambiente, y por ende, en la sociedad de una acción futura llevada a cabo a través de un procedimiento jurídico y administrativo. Es un proceso destinado a mejorar el sistema de toma de decisiones, sobre la base de considerar si los proyectos, programas o políticas pretendidos, resultan ambientalmente sustentables.

El procedimiento abarca desde la presentación de un proyecto por su proponente hasta la declaración, por parte de la autoridad ambiental, de los impactos ambientales de la actividad propuesta.

Luego, otra herramienta muy novedosa que ha sido incorporada por la presente normativa es la de la Audiencia Pública. Esta consiste en la realización de oportunidades de encuentro entre ciudadanos, y quienes tienen la responsabilidad de tomar decisiones. Su finalidad es que las personas se involucren de manera directa, en aquellas decisiones susceptibles de afectarlas. Esta institución es de carácter no vinculante.

Se implementó el Registro Nacional de Infractores que será administrado por la Autoridad Nacional de Aplicación, para que a través de la fiscalización por parte de esta última pueda asegurarse la aplicación de la presente ley, y controlar las condiciones en base a las cuales se otorgaron las autorizaciones de desmonte o manejo sostenible de bosques nativos. Además, en caso de que una determinada persona (sea física o jurídica) cometa alguna conducta ilegal, se establecieron sanciones específicas.

\subsubsection{PARAGUAY}

La ley $N^{\circ} 3001$ de 2006 (de valoración y retribución de los servicios ambientales), surgió como complemento de la ley $\mathrm{N}^{\circ} 422 / 73$ de manejo racional de bosques y tierras forestales. 
Recién en el mes de junio de 2013 (mediante el dictado del decreto $\mathrm{N}^{\circ} 11.202$ ) se reglamentó parcialmente la ley $\mathrm{N}^{\circ} 3001 / 06$, y se establecieron los mecanismos para seguir avanzando en la reglamentación de la misma. Por lo que desde el año 2006 hasta la actualidad, dicha norma fundamental no pudo ser aplicada adecuadamente en la práctica ya que faltaba esta etapa legislativa.

Por su parte, la ley $\mathrm{N}^{\circ} 3001$ tiene como objetivo propiciar la conservación, la protección, la recuperación y el desarrollo sustentable de la diversidad biológica y de los recursos naturales del país, a través de la valoración y retribución justa, oportuna y adecuada de los servicios ambientales.

Asimismo, intenta contribuir al cumplimiento de las obligaciones internacionales que la República del Paraguay ha asumido a través de la firma de instrumentos vinculantes para la protección del medioambiente.

$\mathrm{Su}$ objetivo es similar a la de todas las normas regionales, salvo cuando se refiere a la valoración y retribución justa, oportuna y adecuada de los beneficiarios de los servicios ambientales (vocablos que son vagos y que no han sido adecuadamente definidos).

Además, incorpora el aspecto de la valoración y retribución económica, que resulta muy difícil imponerlos de manera justa para todos los actores sociales, ya que cada uno posee parámetros diferentes.

En el artículo 2 establece que los servicios ambientales son los generados por las actividades humanas de manejo, conservación y recuperación de las funciones del ecosistema que benefician en forma directa o indirecta a las poblaciones, dándole un lugar especial al comportamiento humano.

Los beneficios de los servicios ambientales pueden ser económicos, ecológicos o socioculturales e inciden directamente en la protección y el mejoramiento del medioambiente, propiciando una mejor calidad de vida de los habitantes.

Incluye el stock de capital natural, que combinado con los servicios de manufactura y humano, producen beneficios en las personas.

Esta definición es muy amplia y novedosa, ya que incluye también a la categoría de los bienes naturales ("semi" naturales) en donde el hombre ha intervenido y los ha modificado.

A su vez, aporta una clasificación de servicios ambientales que tampoco fue incluida en otras legislaciones regionales (puntualmente en el caso del inciso a). Esta se vincula con otros recursos naturales diferentes de los bosques y son los siguientes:

a) servicios ambientales relacionados con la mitigación de las emisiones de gases de efecto invernadero: fijación, reducción, secuestro, almacenamiento y absorción de carbono y otros gases de efecto invernadero.

b) servicios ambientales de protección de los recursos hídricos para diferentes modalidades de uso (energético, industrial, turístico, doméstico, riego, etc.) y sus elementos conexos (acuíferos, manantiales, fuentes de agua en general, humedales, protección y recuperación de cuencas y microcuencas, arborización, etc.); 
c) servicios ambientales relacionados con la protección y uso sostenible de la biodiversidad: protección de especies, ecosistemas y formas de vida; acceso a elementos de biodiversidad para fines científicos y comerciales;

d) servicios ambientales de belleza escénica derivados de la presencia de los bosques y paisajes naturales y de la existencia de elementos de biodiversidad y áreas silvestres protegidas, sean estatales o privadas, debidamente declaradas como tales; $\mathrm{y}$,

e) servicios ambientales de protección y recuperación de suelos, y de mitigación de daños provocados por fenómenos naturales.

En el artículo 3 se crea un "régimen de servicios ambientales", cuyo objetivo es establecer un mecanismo técnico y administrativo que permita la valoración o tasación integral de los diversos servicios ambientales brindados por un terreno o finca, y su retribución conforme con éstos.

Los criterios para tener en cuenta en la definición de este régimen son los siguientes:

a) incluirá los diferentes tipos y modalidades de servicios ambientales identificados;

b) incluirá los diferentes participantes (oferentes, demandantes, usuarios);

c) determinará los mecanismos para la definición de políticas, planes y estrategias nacionales en materia de servicios ambientales;

d) desarrollará los criterios técnicos y de zonificación para la valoración integral y retribución;

e) identificación de mecanismos para la definición de prioridades nacionales de inversión en retribución por servicios ambientales;

f) identificación de los mecanismos de administración para la captación y distribución de los ingresos provenientes de los servicios ambientales; $y$,

g) mecanismos de monitoreo y auditoría para la verificación del adecuado uso de los recursos.

Se exige muy acertadamente que los oferentes que deseen ingresar al régimen de servicios ambientales, cuenten con una declaración de impacto ambiental. 
La certificación que se hubiera expedido por aquellos servicios ambientales generados por las actividades de manejo, conservación y recuperación de los recursos naturales que realizara el titular de los mismos no podrá ser menor a cinco años.

Además, los propietarios o poseedores de elementos de la naturaleza que contribuyan a la generación de servicios ambientales, tendrán derecho a la correspondiente retribución por los servicios prestados. Para ello, el Estado definirá lineamientos para la fijación de los valores de dichos servicios.

Además, el Poder Ejecutivo deberá definir cada año la "lista de los servicios ambientales" reconocidos, y los montos correspondientes a su retribución, dependiendo de la naturaleza de los mismos. El régimen es taxativo, lo cual no resulta favorable en la práctica ya que es de carácter rígido.

Los servicios ambientales ya reconocidos mantendrán su validez por el tiempo que esté reconocido en la certificación respectiva, salvo que se produzcan violaciones a la misma, lo cual hará decaer automáticamente los derechos que le fueran inherentes a las personas.

Se establece que el Poder Ejecutivo establecerá el valor de los servicios ambientales, el que será actualizado cada cinco años, sin perjuicio del establecimiento de un índice de ajuste de precios para mantener dicho valor entre cada nueva valorización. Esta postura se dificulta en países donde las tasas de inflación son altas.

Su precio inicial será establecido en relación con el valor o beneficio económico, ambiental o sociocultural que satisfaga.

Se emitirá un certificado de servicios ambientales, a ser obtenido por personas físicas o jurídicas que, en virtud del proyecto que vayan a ejecutar o la actividad que realicen, estén obligadas a invertir en servicios ambientales; así como por cualquier otra persona física o jurídica, nacional o extranjera que tenga interés en prestar dichos servicios o a pagar para que un tercero lo preste.

El certificado de servicios ambientales es un título valor libremente negociable por quienes no están obligados en virtud de esta ley o por sentencia judicial a invertir en servicios ambientales, y podrán negociarse en el mercado internacional para el pago de compensaciones medioambientales efectuadas por las personas físicas o jurídicas obligadas al efecto por las actividades o explotaciones que realicen y que sean consideradas nocivas para el ambiente.

Se deberá mantener y garantizar la relación entre la cantidad y calidad de los recursos naturales incluidos en el régimen de servicios ambientales y los certificados de servicios ambientales vigentes y en circulación.

\subsubsection{PERÚ}

En este caso, el Poder Ejecutivo solicitó en varias oportunidades al Congreso que apruebe el proyecto de ley para la compensación de los servicios ambientales de 2009, cuyo objeto es establecer el marco general para la compensación y/o retribución de los servicios ambientales con la finalidad de coadyuvar a la conservación, recuperación y uso sostenible de la diversidad biológica y de los recursos naturales del país. 
A diferencia de lo que ocurre con la normativa de Paraguay, aquí no se realiza una valoración justa y equitativa (previa) a la compensación y/o retribución de los servicios ambientales, lo cual supone que todo quedará a discreción del Estado.

En la exposición de motivos del proyecto de ley se han establecido diversos criterios que resultan interesantes para comprender el espíritu de la norma.

Se establece que al igual que ocurre con el caso de Argentina, Perú contaba con una ley general del ambiente, que estableció un marco para el tratamiento de los servicios ambientales, no habiendo sido promovidos los mecanismos de financiamiento y pago de los mismos.

Se reconoce la existencia de un vacío legal (tema que todavía no ha sido reconocido por Argentina ni incluido en su agenda nacional), alegando que esto impactaba en la gestión y protección de los ecosistemas boscosos, los que además de brindar una serie de productos forestales maderables y no maderables, suministran también servicios ambientales como: provisión de agua, captura y almacenamiento de carbono, estabilidad de laderas, provisión de principios activos y sustancias para la prospección biológica, belleza escénica, entre otros.

Se destacaron los compromisos legales internacionales adquiridos por Perú, como parte del Acuerdo de Promoción Comercial Perú - Estados Unidos y su Protocolo de Enmienda, donde se comprometió a implementar en su legislación y propiciar el fortalecimiento institucional, y que mejore las condiciones de competitividad económica para el óptimo aprovechamiento de los servicios ambientales.

Se precisa que la elaboración del inventario de servicios ambientales y los mecanismos para valorizar, retribuir, mantener la provisión de los mismos y la promoción de su pago está a cargo del Ministerio del Ambiente.

Igualmente, se establece la compensación por servicios ambientales como un instrumento de gestión que coadyuva al uso sostenible y conservación de los ecosistemas y recursos naturales.

Se establece que el Ministerio del Ambiente es la autoridad competente para la promoción, regulación y supervisión de las iniciativas, proyectos y programas de compensación por servicios ambientales. Este aspecto es fundamental para asegurarse que su aplicación sea lo más acorde con el texto de la norma, ya que debe contarse con un adecuado apoyo estatal a través del funcionamiento y la provisión de los recursos necesarios (humanos, económicos, políticos).

En diversos países de la región (como es el caso de Argentina cuya gestión la realiza una Secretaría), no poseen una estructura ministerial para la protección del medioambiente, lo cual dificulta su aplicación.

Luego, en el artículo 2 se define a los servicios ambientales como aquellos resultantes de funciones y/o procesos ecológicos de ecosistemas o recursos naturales, que generan beneficios económicos, sociales y ambientales a la sociedad.

Se entiende como servicios ambientales a los siguientes:

a) La protección y conservación de fuentes de agua y cuencas hidrográficas; 
b) La protección y conservación de las cuencas atmosféricas;

c) La protección, conservación y recuperación del suelo;

d) La protección o conservación de la biodiversidad, especies y ecosistemas;

e) La mitigación de emisiones y la fijación, reducción, secuestro, almacenamiento y absorción de gases de efecto invernadero;

f) La belleza escénica;

g) La asimilación y diseminación de efluentes y emisiones, y

h) En general, el mantenimiento de los procesos ecológicos esenciales de los ecosistemas naturales.

Aquí también, a diferencia de lo que ocurre con la legislación de Paraguay, no se incluyen a los aportes humanos en la generación de servicios ambientales, sino que se los define únicamente teniendo en cuenta la características naturales del entorno. Esta postura se revierte en el artículo 3 cuando se menciona que en determinados casos pueden considerarse a las intervenciones humanas, como fuentes de servicios ambientales.

Se establece que la compensación por servicios ambientales es un mecanismo flexible y promisorio que contribuye al uso sostenible y a la conservación de los ecosistemas y recursos naturales.

Sus finalidades son las siguientes:

a. Procurar un retorno a las comunidades y personas que apoyan o mantienen las fuentes $\mathrm{y} / \mathrm{o}$ patrimonio natural que prestan el servicio ambiental;

b. Conservar la fuente $y / o$ patrimonio natural;

c. Estimular la participación privada en la conservación de la fuente y/o patrimonio natural

Se estableció que el Estado debe evaluar y preservar el patrimonio natural que es fuente de los servicios ambientales.

El Ministerio del Ambiente como autoridad competente debe promover y regular la inversión pública y privada, y otros instrumentos económicos relacionados a servicios ambientales y elaborar el inventario de servicios ambientales, establecer los mecanismos para valorizar, retribuir y mantener su provisión, y aprobar las iniciativas de compensación por servicios ambientales y las registra. 
Además, debe promover el uso de valores negociables del sistema financiero nacional e internacional a través, entre otros, de certificados de servicios ambientales y cualquier otro tipo de instrumentos de renta fija y/o variable, que permitan la financiación de proyectos de servicios ambientales y demás actividades relacionadas con ellos que precise el reglamento.

\subsubsection{COLOMBIA}

En el mes de mayo de 2012, el Ministerio del Medio Ambiente publicó el decreto $\mathrm{N}^{\circ} 953$ que presenta las condiciones para que los entes territoriales (departamentos y municipios) financien el pago por servicios ambientales, y realicen la adquisición de predios en áreas de importancia estratégica para la conservación del recurso hídrico.

Mediante este decreto, se le reconoce un derecho esencial a los propietarios de predios ubicados en áreas de conservación de cuencas hidrográficas, que dediquen parte de su propiedad a la conservación y recuperación de los ecosistemas naturales. Como incentivo y compensación, los propietarios reciben el equivalente al costo de oportunidad por no usar dichas zonas en otras actividades productivas propias de su región.

En Colombia, según la ley para la conservación y recuperación de las cuencas, los departamentos y municipios deben asignar por lo menos el $1 \%$ de sus ingresos para la adquisición de predios o el pago por servicios ambientales.

El decreto señala que las autoridades ambientales, previamente, deberán identificar, delimitar y priorizar las áreas de importancia estratégica y luego monitorear su conservación.

En su artículo 8 dice: "El incentivo de pago por servicios ambientales aplicará transitoriamente mientras la entidad territorial adquiere el respectivo predio localizado en dichas áreas".

Esto puede interpretarse como si una vez ingresado al sistema de pago por servicios ambientales el propietario compromete su título, ya que a mediano plazo su predio sería adquirido por el ente territorial.

El artículo 9 (parágrafo 2), al hacer referencia a la formalización de acuerdos y el registro de pago por servicios ambientales, dice: "La aplicación del incentivo no conlleva pronunciamiento alguno acerca de la titularidad de los derechos reales ni tiene efecto en relación con la propiedad o la tenencia de la tierra o adquisición de derechos". Esto significa que recibir pago por servicios ambientales no compromete la venta posterior de la tierra al ente territorial.

\subsection{PROPUESTAS PARA OBTENER UNA GESTIÓN MÁS SUSTENTABLE DE LOS SERVICIOS AMBIENTALES}

\section{a) La educación ambiental}

La educación ambiental (que es uno de los principios fundamentales del Derecho Ambiental) en relación a los aspectos de protección, conservación, utilización 
razonable y no contaminación de los recursos naturales, podría apoyar en la práctica a las herramientas legales existentes tanto a nivel nacional como internacional.

En todos los países analizados, la mayoría de los propietarios de tierras están en conocimiento de las leyes, pero no las cumplen por ausencia del Estado o de mecanismos legales para su aplicación.

Coincidimos con algunos autores que afirman que "precisamente la educación ambiental es la única capaz de construir la conciencia colectiva sobre el valor del ambiente y la necesidad de cuidarlo y recuperarlo." (Mathus Escorihuela, 2011).

En este sentido, la falta de capacitación y educación ambiental de las comunidades locales, ha sido una de las debilidades detectadas en las diferentes regiones analizadas. La escasez de directrices en ese sentido, acompañado por el bajo presupuesto asignado ha maximizado la problemática, hasta tal punto que se desconocen los lineamientos centrales y la existencia de normativa vigente.

Muchas veces, esto sucede porque no hubo una tarea previa de concienciación dirigida hacia los alumnos y docentes.

En Argentina debe destacarse que existe normativa vigente que califican a la Educación Ambiental como fundamental, como es el caso de la Constitución de la Provincia de Buenos Aires (artículo 200), la ley nacional $\mathrm{N}^{\circ} 25.675$, y la ley provincial (Buenos Aires) $\mathrm{N}^{\circ} 11.723$.

A su vez, en el ámbito educativo nacional no se ha incorporado la asignatura "Educación Ambiental" como obligatoria dentro de la currícula oficial, por lo que tampoco existen muchos antecedentes de producción de material vinculado con la misma (salvo los programas contemplados por la Secretaría de Ambiente y Desarrollo Sustentable de la Nación pero todavía muchos de los cuales no han sido implementados).

Tanto el público, las $\mathrm{ONG}^{\prime}$, las instituciones educativas, los medios de comunicación y la población en general tienen un rol muy importante respecto de la difusión y educación en dicha área. Todos ellos colectivamente deben imponer presión para tender a lograr dicho objetivo (Nelliyat, Prakash; Ambujan, 2011).

A su vez, cuando nos referimos a niveles superiores de educación o de investigación en dicha área, la tendencia general es la escasa transferencia de conocimientos desde la comunidad científica hacia la sociedad.

Actualmente en Argentina se ha dictado la ley de libre acceso a la información científica, que establece que las instituciones del Sistema Nacional de Ciencia y Tecnología y que reciban financiamiento del Estado Nacional, deben crear repositorios digitales institucionales de acceso abierto y gratuito en los que se depositará la producción científico tecnológica nacional.

La producción científica que será publicada en los repositorios digitales abarca trabajos técnico-científicos, tesis académicas, artículos de revistas, entre otros; que sean resultado de la realización de actividades de investigación financiadas con fondos públicos ya sea, a través de sus investigadores, tecnólogos, docentes, becarios postdoctorales y estudiantes de maestría y doctorado. La ley establece además la obligatoriedad de publicar los datos de investigación primarios luego de 5 años de su recolección para que puedan ser utilizados por otros investigadores. 
Un aporte importante podría ser el de "celebrar acuerdos institucionales con sectores académicos (Universidades, ONGs, escuelas técnicas, instituciones públicas de ciencia y técnica, etc.) tendientes a aportar, desde sus distintos ámbitos, elementos de juicio y de seguimiento analítico (independiente) respecto a la amplia gama de dimensiones que involucra la prestación del servicio de aguas y saneamiento." (Azpiazu, Catenazzi, Forcinito, 2004).

\section{b) Veedurías ciudadanas}

Estas fueron definidas como una herramienta de vigilancia y control social de la ciudadanía sobre el Estado, para fortalecer la vigencia de los principios constitucionales tales como democracia, transparencia, ética, equidad, eficiencia, y eficacia, en la adopción e implementación de políticas públicas, en el desempeño de responsabilidades de los funcionarios públicos y en la gestión pública en general.

Las veedurías están conformadas por ciudadanos honestos encargados de vigilar la gestión de los funcionarios e instituciones del Estado para prevenir los malos manejos del sector público (Comisión de control cívico de la corrupción, 2003). Básicamente, controlan el funcionamiento de los organismos de control en Ecuador (UNDP, 2011).

La vigilancia ciudadana es aquella actividad en la que las personas están pendientes de la actuación y del cumplimiento de responsabilidades de funcionarios, funcionarias y autoridades a cargo de la gestión pública, así como de los procesos implantados por ellos mismos (Comisión de control cívico de la corrupción, 2003).

Cabe destacar que una de las principales áreas que se autoriza el control a las veedurías ciudadanas son los programas vinculados con el medioambiente, por lo que los servicios ambientales podrían adecuadamente ser directamente monitoreados.

Existe una clasificación de veedurías:

a) Pequeñas y medianas vinculadas con programas, proyectos y en general, a temas vinculados a la administración pública en los ámbitos local, municipal y sectorial.

b) Veedurías vinculadas con programas y proyectos nacionales cuyo grado de afectación al interés público requiere de vigilancia ciudadana.

c) Veedurías vinculadas con aspectos generales de interés público que presenten indicios de corrupción (Comisión de control cívico de la corrupción, 2003).

\section{CONSIDERACIONES FINALES}

Finalmente, podemos concluir que existe una disparidad normativa en relación con la normativa sobre servicios ambientales de los países de América del Sur que fueron analizados. 
En primer lugar, tanto Argentina como Colombia no poseen una ley de servicios ambientales y se rigen por una norma protectora de un recurso natural específico (como es el bosque nativo o del medioambiente). En este caso faltaría un desarrollo normativo que regule todos los aspectos de los servicios ambientales.

En segundo lugar, Paraguay y Perú poseen normas que regulan especialmente a los servicios ambientales, la primera ha marcado una notable calidad normativa. En el caso de Perú su proyecto de ley está próximo a aprobarse por el Congreso.

Estos podrían ser tomados como base para la elaboración de futuros proyectos de leyes, pero teniendo algunas consideraciones particulares, e incorporando las dos herramientas propuestas para lograr una gestión más sustentable de los servicios ambientales.

Esto es así porque "los trasplantes de esquemas o instrumentos jurídicos de salvaguarda de la biodiversidad de un país a otro, por lo mismo, deben efectuarse muy cuidadosamente y sin perder de vista que lo que bajo ciertas matrices culturales puede ser apropiado y beneficioso para una nación, puede no serlo para otra, cuyo escenario jurídico se plantea y discurre sobre la base de categorías valorativas diferentes." (Valenzuela Fuenzalida, 2010).

La disparidad normativa va en contra del espíritu del bloque regional del MERCOUSR al que pertenecen Argentina y Paraguay, y cuyo objetivo es unificar el marco jurídico ambiental en la región, según dimana de la Resolución $\mathrm{N}^{\circ} 22 / 92$ del Grupo de Mercado Común del MERCOSUR, en donde se establecieron los siguientes objetivos:

a. Analizar la legislación vigente de cada Estado parte y sacar conclusiones acerca de sus asimetrías políticas y legislativas en materia ambiental.

b. Elaborar una propuesta de directrices básicas en materia de política ambiental.

Para los casos de los países en donde existe un vacío legal respecto de la regulación de los servicios ambientales, debería preverse normativa que sea acompañada de políticas públicas tendientes a lograr dos objetivos:

a. La protección de los recursos hídricos y de su calidad.

b. Asegurar la infraestructura y las inversiones necesarias para poder absorber adecuadamente las nuevas demandas de los usuarios.

El Derecho Ambiental debería adoptar un mecanismo técnico que internalice el cobro por servicios ambientales brindado por todos los recursos naturales.

Sin perjuicio del innegable progreso que significa contar con estas normas, todavía el grado de implementación de la legislación ambiental en general es bajo. Se requiere todavía un mayor grado de institucionalización y organización de estructuras públicas que puedan hacer frente a las dificultades que presentan las cuestiones ambientales. 
No es suficiente contar con leyes, tratados, estatutos o reglamentos muy bien redactados por especialistas y es fundamental que tales normas surjan de acuerdos previos sobre cuáles son los problemas y cómo se piensa encararlos (Pochat, 2005).

Debe tenerse en cuenta y evitar lo establecido desde el área de la Sociología Jurídica, en relación a que la norma escrita tuvo en el modelo típico ideal la función de asegurar la seguridad de los mandatos, y ha llevado como disfunción (o corrupción), a generar una inmanejable proliferación normativa (Fucito, 2003).

\section{BIBLIOGRAFÍA}

AZPIAZU, D.; CATENAZZI, A.; FORCINITO, K. (2004). Recursos públicos, negocios privados, Informe de Investigación $\mathrm{N}^{\circ} 19,2^{\circ}$ edición, Universidad Nacional de General Sarmiento, ISBN: 987-9300-59-9, Los Polvorines, Buenos Aires, Argentina.

COMISIÓN DE CONTROL CÍVICO DE LA CORRUPCIÓN (2003). Manual de veedurías ciudadanas, Ecuador, páginas 15, 21, 23, ISBN 9978-92-280-6.

FUCITO, F. (2003). Sociología del Derecho, Editorial Universidad, $2^{\mathrm{a}}$ edición, ISBN: 950-679-330-1, página 458, Buenos Aires, Argentina.

HANTKE-DOMAS, M.; JOURAVLEX, A. (2011). Lineamientos de política pública para el sector de agua y saneamiento, Documento de proyecto, página 23, CEPAL, Santiago de Chile.

INTERNATIONAL UNION FOR CONSERVATION OF NATURE (IUCN) (2009). RULE - Reforming water governance, páginas 59, 65, Gland, Suiza, ISBN: 978-28317-1027-3.

INTERNATIONAL UNION FOR CONSERVATION OF NATURE (IUCN) (2010). Pagos por servicios ambientales: marcos jurídicos e institucionales en IUCN Serie de Política y Derecho Ambiental N ${ }^{\circ} 78$, página 21, 22 y 23, Thomas Greiber Editor, 978-2-8317-1177-5, http://data.iucn.org/dbtw-wpd/edocs/EPLP-078-Es.pdf.

MATHUS ESCORIHUELA, M. (2011). Sinergias ambientales e instituciones de gestión en libro Gobernanza y manejo sustentable del agua, Editorial Mnemosune, Editora Griselda Capaldo, ISBN; 978-987-1829-03-3, Buenos Aires, Argentina.

MINAVERRY, C.; GALLY, T. (2012). La implementación de la protección legal de los bosques nativos en la Argentina en Revista Pensamiento Jurídico $\mathrm{N}^{\circ} 35$, páginas 253-278, Editorial de la Facultad de Derecho, Ciencias Políticas y Sociales, Universidad Nacional de Colombia, ISSN: 0122-1108.

MINAVERRY, C. (2011). Fortalezas y debilidades de la legislación sobre protección de bosques nativos, y su vinculación con la normativa sobre aguas en la Argentina, en: Memoria del Primer Encuentro Internacional de Derecho Forestal Ambiental del Cono Sur, 7 al 10 de Septiembre de 2011, IUFRO, Asunción Paraguay, pp 4.

NELLIYAT, PRAKASH, A. N. (2011). Need for Integrated Water and Land Resources Management. Approach for Sustainable Ecosystem and Water Security in Third World Cities en Abstract Volume, World Water Week in Stockholm, Stockholm International Water Institute (SIWI), Editora: Ingrid Stangberg. 
PAGIOLA, S.; PLATAIS, G. (2002). Pagos por servicios ambientales en revista Environment Strategy $\mathrm{N}^{\circ} 3$, página 3.

POCHAT, V. (2005). Entidades de gestión del agua a nivel de cuencas: experiencia de Argentina en Serie de Recursos naturales e infraestructura, $N^{\circ}$ 96, CEPAL, página 49, Santiago de Chile.

ROUSE, M.(2007). Institutional Governance and Regulation of Water Services, International Water Association (IWA), ISBN: 1843391341, páginas 20 y 27, Londres, Reino Unido.

TEXTOS NORMATIVOS regulando a los servicios ambientales de Argentina, Perú, Paraguay y Colombia.

UNITED NATIONS DEVELOPMENT PROGRAMME, UNDP (2011). Informe de lucha contra la corrupción en el sector del agua, Métodos, herramientas y buenas prácticas, página 57, Nueva York.

VALENZUELA FUENZALIDA, R. (2010). El Derecho Ambiental. Presente y pasado, Editorial Jurídica de Chile, Santiago de Chile, Chile, ISBN: 978-956-10$1982-9$ 